\title{
Is Virtual Reality the Future of Learning? A Critical Reflection
}

\author{
Anna Schwarze \\ University of Siegen \\ anna.schwarze@uni- \\ siegen.de
}

\author{
Henrik Kampling \\ University of Siegen \\ henrik.kampling@uni- \\ siegen.de
}

\author{
Oliver Heger \\ University of Siegen \\ oliver.heger@uni- \\ siegen.de
}

\author{
Bjoern Niehaves \\ University of Siegen \\ bjoern.niehaves@uni- \\ siegen.de
}

\begin{abstract}
The year 2016 marks the so-called second wave of VR, which was initiated by the first consumer VR-HMD, Oculus Rift (development kit), entering the market. There are four practical advantages in the field of virtual reality learning: a shift from abstract to tangible settings, interactivity rather than passive observations, using desirable but practically infeasible methods, and breaking the bounds of reality. In contrast, current VR technologies also feature certain limitations. The most common negative factor is motion sickness, which distracts the user. We conducted a multiple case study and invited 41 people to participate in two different scenarios. One was a self-developed $360^{\circ}$ video and the other was a selfdeveloped interactive scenario. We investigate different barriers which hamper individual learning in $V R$ and we point out that there is a potential for implicit learning in virtual reality.
\end{abstract}

\section{Introduction}

Virtual Reality (VR) based on head-mounted displays (HMDs) offers the possibility to design an individual immersive environment. HMDs, such as Oculus Rift or HTC Vive, have generated a new hype around immersive VR systems, especially in the gaming industry since the Oculus Rift development kit entered the market in 2016 [13]. Apart from gaming, more and more sectors make use of VR technology, such as healthcare or education [48]. VR-HMD sets itself apart because it creates immersive experiences by immersing its user in a digital environment. Psychological immersion is "the mental state of being completely absorbed or engaged with something" [8]. From a technological perspective, immersion means "the experience of total engagement where other attentional demands are, in essence, ignored" [1].

Given this special characteristic, VR can be a powerful tool to design learning environments to improve individual learning performance. This can be explained by the theoretical construct of immersion as part of cognitive absorption (CA). CA is defined as a deep involvement with technology [1] and is based on the concept of flow, which is described as a mental state of absorption, a feeling of engagement (such as concentration), a sense of being in control, a loss of self-consciousness, and a shift in perception of time [4]. Flow is an important concept in the context of e-learning. It generates positive effects for the learner [37]. VR has the potential to create an immersive learning environment inducing a state of high flow to improve learning outcomes [28].

However, VR-HMD technology that is currently available on the consumer market comes with certain limitations. One constraint is motion sickness [29]. Typical symptoms of motion sickness are pallor, sweating, and nausea $[15,17,22,41]$, eyestrain, vomiting, fatigue, disorientation, or dizziness [15, 17]. As a consequence, current VR technology can only be used for a few minutes until it causes discomfort. Obviously, a state of discomfort would decrease learning performance.

So far, there is little systematical or empirical research on drivers and barriers when using VR for learning. According to Slater and Sanchez-Vives [46], VR systems make abstract settings more tangible, enable the learner to be active rather than just a passive observer, enable the user to use methods that are desirable but practically infeasible even if possible in reality, and allow users to break the bounds of reality to explore different options. Yet, it remains unclear how VR-HMD can be used appropriately for learning purposes.

We therefore aim to explore advantages and disadvantages of VR-HMD and to point out how current VR technology can potentially improve individual learning. Our research question is:

RQ: What are drivers and barriers of VR-HMDs in the context of individual learning?

The study at hand uses a multiple case study approach. Case studies can record different phenomena in relation to their context [18], which is the objective of our research. We use literature on VR and individual learning and carry out a pilot study to gain first insights 
into the subject. After that, we develop and carry out two case studies in which the participants experience a HTC Vive and a self-developed application for learning first-hand. We present and discuss the implications of our findings and make propositions on learning in VR.

\section{Related Work}

Virtual reality and learning. Over the course of the past few years, we have seen the rise of the socalled second wave of VR [2]. During that time, the first consumer oriented VR-HMD, i.e. Oculus Rift, has entered the market and others, such as HTC Vive, PlayStation VR, or Samsung Gear VR [2], have followed. VR features different characteristics and provides its user with an interactive, computergenerated, and three-dimensional virtual space [52]. There are two different kinds of VR: immersive and non-immersive VR [28, 50]. Non-immersive VR refers to personal computers with a screen in front of the user, while immersive VR refers to the idea that a user can enter a totally immersive and enclosed virtual space by using a VR-HMD [43]. Immersion is defined as "a mental state of being completely absorbed or engaged with something" [8:3]. According to Slater, users become immersed in an environment that completely surrounds them while wearing an HMD that simulates movements and motion parallax when they turn their heads [47]. VR mostly refers to a singleuser interaction in a virtual environment and is typically limited to sessions of 30 minutes [42]. VR users are provided with controllers to interact, create, or manipulate objects within the virtual space [19, 44]. In order to generate an immersive experience during a VR session, VR sound is also relevant [11]. An overview of current VR technologies shows diverse hardware devices featuring visualization, haptic, or multi-sensor and software applications such as game engines and open platforms [2]. Another advantage of virtual environments is the precision to which objects and processes that are abstract, difficult, or impossible to depict in the real world are visualized [27, 46]. These characteristics encourage focused experiences, such as for different learning contexts. A review article provides an overview of the value of VR systems, publications in the field of education, targeted populations, areas of interest, and to what extent a VR system can be a source of motivation [31]. One example for the latter is gamification. This approach can be used by teachers to motivate their students to better engage in the learning process.

Moreover, four practical advantages have been identified within the field of VR and learning [46]: first, VR systems make abstract settings more tangible. For example, geometrical mathematics can be better understood in VR settings than in the classic paper and pencil setting; or biological cells can be visualized to demonstrate how they work [21]. Second, VR enables the learner to be active rather than just act as a passive observer. A quantitative study concludes that VR medical training, notably in surgery, benefits immensely from active interactions combined with haptic feedback [30]. Third, VR enables its user to use methods that are desirable but practically infeasible even if accessible in reality. For instance, if a teacher wants to visit historically important places around the world, such as Niagara Falls in week one, Grand Canyon in week two, Stonehenge in week three (and so on), this will (probably) be practically infeasible because of time and other restrictions on resources. VR offers the opportunity to visit all these places in a short period of time [24]. Fourth, VR allows its users to break the bounds of reality to explore different realms. For example, it would be interesting to explore what happens if gravity changes while someone is juggling or what it means to change the speed of light (what are the effects and what would it mean to humanity) [8].

Current research on VR environments follows different approaches to examine learning and task performances. Almost all of them base their research on immersion itself or on immersion as part of cognitive absorption [1]. In an online mobile training scenario, perceived individual learning is investigated in case of a user's deep involvement with a task [35] and individual learning outcomes are examined in peer influenced learning with groups through the medium of text and video [36]. Within a multi-user online virtual environment, a quantitative study shows that context and social facilitation as well as immersion influence learning while working on collaborative tasks [14]. Another study focusing on collaborative tasks examines the impact of group learning behavior and immersion on individual learning in an organizational context [26]. Additionally, another study examines the effect of social presence, interest, and immersion on learning in different contexts, such as satisfaction in online environments [23].

Current limitations on learning in VR. A consistent issue and drawback of the use of VR technology is cyber sickness and its manifesting symptoms of motion sickness [44]. Motion sickness occurs when the body of a person is physically stationary while the view of an individual still conveys that they are in fact moving [22]. As a consequence, the person's brain receives different types of sensory input. The most common forms of motions sickness are sea and air sickness. With the emergence of VR, the issue of motion sickness has been increasing 
significantly, notably through visual illusions and selfmotion [15]. Motion sickness typically manifests itself in two types of situations: 1) when there are excessive lags between the presented visualization on the visual head-mounted display and the head movements of the individual, and 2) when the head movement of the individual and the movements in virtual reality do not match in real-time [15].

All of these factors can lead to accidents in the virtual environment which, in turn, affects reality [17]. In the context of virtual reality, there appear to be additional potential side effects. For instance, there is a range of reports of flash-backs which produce problems while driving [45]. Overall, motion sickness negatively affects VR experiences, especially in the case of HMDs, because it has been hindering the adoption and production of VR technologies. Moreover, motion sickness can disturb the immersion of its user [41] and, hence, it prevents an individual from focusing on the learning context or from performing a certain task.

In order to answer our research question, we have just identified related work on VR in the context of learning. Here, many potential advantages, such as immersion (a state of total engagement), interactive scenarios, depiction of abstract elements, motivators, haptic feedback through provided controllers, substitution of practically infeasible methods, or breaking the bounds of reality, have been pointed out. However, there is only little research that focuses on the negative side of using VR systems for learning. Motion sickness is the aspect that is examined the most and that appears to be a main drawback of VR technology. Nevertheless, we assume more research is needed to focus on current barriers of using VR technology for learning contexts. Hence, our study aims to explore drivers as well as barriers of VR technology in learning contexts.

\section{Method}

Method selection. We have chosen a multiple case study in order to examine individual learning in the context of virtual reality [53]. Case study research is particularly appropriate for complex contexts which have not yet been fully explored $[3,18,53]$. Moreover, case studies allow an in-depth exploration of different phenomena and the context in which they occur [18]. Both aspects are relevant to our study.

A team of at least two researchers (the first and second author, the third author gave additional support during the matching phase) conducted this study, which should reduce idiosyncratic perceptions. By involving multiple researchers we were able to establish triangulations, i.e. investigator triangulation [32]. In order to reduce case-related findings, we chose a heterogeneous group of respondents [3, 10, 53]. Generally, it is assumed that the significance of a case study relies on its internal validity, whereas the external validity is considered a weakness.

Case study design. The aim of this study is to examine the use of immersive VR environments for individual learning. To answer our research question, we focus on how and in which context a learner would like to use an immersive VR application and possible positive and negative factors. Therefore, the unit of analysis is an individual (a person; [3]). Additionally, this unit of analysis includes multiple levels of analysis (i.e. diverse interviewees) and different cases $\left(360^{\circ} \mathrm{VR}\right.$ videos and interactive VR scenario). Therefore, we are able to strengthen our findings in terms of replication logic $[10,53]$. This case study consists of four phases (see figure 1), which are briefly described below: pilot study, case selection, data collection, and data analysis.

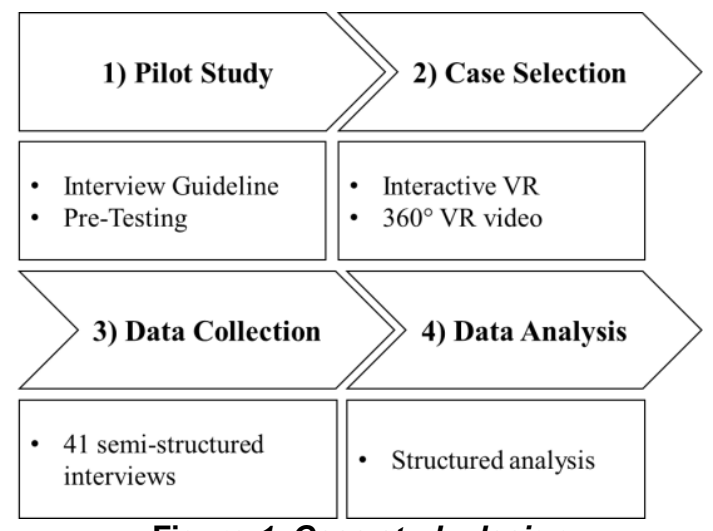

Figure 1. Case study design

Pilot study. First, we developed two different interview guidelines and conducted a pilot study to ensure that our questions were clear. We recruited two employees of a medium-sized university for each interview guideline. The employees were provided with a self-developed, immersive VR application (we used the HTC Vive hardware) to gain first-hand experience. After the respective interviews, the researchers carefully read the transcripts and reformulated incomprehensible questions.

Case selection. Based on the pilot study, we recruited further potential users for the two different immersive VR applications. We looked for people with different professional and demographic backgrounds as decision criteria. For the first study, we were looking for people from the vocational school context in particular. Here, we were able to recruit people from the vocation school apprenticeship sector, trainees, and students studying to become teachers for vocational schools, but also people with a consulting background. 
For the second study, we recruited people from the education, crafts, architecture, and business sector. Each of the interview participants confirmed that they had experience in learning but not with immersive VR technology in advance. In the first case study, we used a self-developed program for learning business processes, created with unreal engine 4 . In the second study, we used a $360^{\circ}$ video of a self-developed process based on Minecraft VR.

Data collection. Immersive VR such as HTC Vive or Oculus Rift is a novel type of technology. Before we started the interview, each participant had the opportunity to acquire first-hand experience using VR technology. This first-hand experience and the interview took place in a computer laboratory. This ensured that each of the participants could gain experience in VR and that each participant could answer questions on the VR application. In the second study on motivation in VR, we recruited people with and without experience. People with VR experience had acquired their experiences from a previous study. The interview questions aimed to understand VR-based learning in vocational schools and training as well as presenting motivation opportunities for knowledge acquisition. At the beginning, the interviewees were not informed of the focus of the study so that they kept an open mind. We chose this approach so that the participants would not just focus on one specific aspect of VR. During the interview, the participants were asked about their opinion on what they liked and what they did not like.

In the first study, each participant was introduced to a self-developed immersive VR learning environment (c.f. figure 2.) The interviewee was able to freely move in the virtual room. Here, the starting point was behind a desk with two types of measuring instruments. The task was to sort different kinds of letters and parcels into the correct box. Within the VR environment, participants were able to move by also moving in reality and perform the task by using the provided controllers.

In the second study, the interviewees were introduced to a self-developed $360^{\circ}$ VR video which was developed in Minecraft VR (c.f. figure 3). The participant sat on a real chair and was introduced to the VR and led through the process in a controlled manner. They could watch the virtual video of a brewery process while the system led through the different steps of the beer brewing process. The participant was provided with additional information on the process on info panels. They were able to start/stop the video by using a computer mouse.

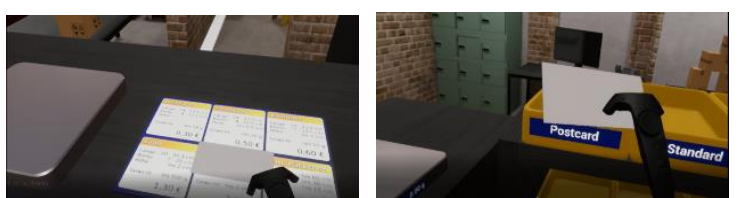

Figure 2. Self-developed VR application

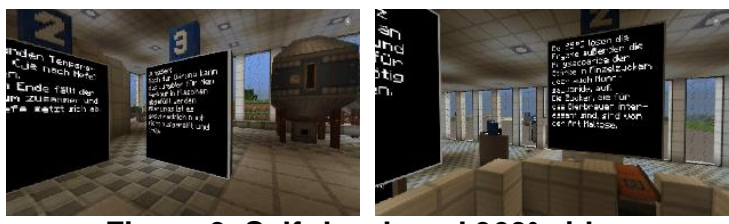

Figure 3. Self-developed $360^{\circ}$ video

On average, the interviews in the first study took 38 minutes. We interviewed 12 female and 19 male persons with an average age of 39 years (see Table 1). Within this interview group, we had seven teachers, a laboratory assistant, an adult pupil, two teachers, an industrial management assistant, a production planner, a metalworker, a student of business and SME management, a student of economics, an occupational therapist, an emergency paramedic, two production mechanics, an electrical engineer, an ERP-Consultant, a management consultant, an innovation consultant, a practice nurse, a research associate, a student of environmental science, two students of IS, and a student of business administration.

Our second study had an average duration of 13 minutes. We interviewed 5 women and 5 men with an average age of 33 years. This interview group consisted of an adult pupil, two educators, a student of IS, two carpenters, an engineer, an economist, an architect, and an unemployed person.

Both studies followed a semi-structured interview guideline. Considering that every interviewee can bring up further connections, which were not included in our interview guideline, each interview was open-ended [6]. While using the VR application, every interviewee was able to ask questions. We conducted the interviews with two interviewers following the guideline of Darke et al. [6]. The interviews were recorded and entirely transcribed to minimize data loss.

Data analysis. In order to analyze our interviews, we used MAXQDA 12. In both studies, we focused on indicators with positive and negative implications of virtual reality technology. In order to analyze the indicators, we applied the grounded theory method [12, 49 , 51] i.e. open coding, axial coding, and selective coding. Therefore, we conducted the study in three phases. For each phase, the first two authors worked independently from each other but collaborated with the third author to execute the coding and codematching in order to reach a joint result. 
In the first phase of coding, i.e. open coding, we searched for emerging aspects by analyzing the interviews line by line. Here, we also made sure that no themes were excluded due to prejudice. In the next step, we analyzed the interviews by axial coding. We built clusters of similar codes and identified different insights that were relevant to our subject. In the third phase, we searched for relations to better understand themes and to draw conclusions by looking for parallels to existing literature (selective coding). After 41 interviews, we finished our data collection because there were no new insights (theoretical saturation). We chose the following quotations because they were most appropriate to represent our findings and structured to present drivers as well as barriers of learning in VR.

\section{Major Findings}

Drivers of learning in VR: Interactivity. The advantages of the interactive scenario (study 1) in comparison to the $360^{\circ}$ video (study 2 ) are being able to move freely in the virtual environment, interactivity because of controllers, and the balance and presence of the participant in the virtual reality provided by the controllers.

"Yes, with the controllers you can actually be active in the virtual room." (I 2, student of teaching, study 1)

"The positive effect of the controllers is that you can interact with the environment." (I 18, student of teaching, study 1)

"The controller has a positive effect because you can interact with it. If you couldn't do that, you'd be standing in an empty room, and then VR wouldn't make sense." (I 7, emergency paramedic, study 1)

Mobility. One advantage of the $360^{\circ}$ video is the possibility of watching it with a smartphone and a cardboard. In this way, you are in the position to take the learning material with you and learn anywhere and anytime you want.

"I use IT-supported learning systems with my smartphone because I take it anywhere with me." (I 2, educator, study 2)

"The mobile version can be taken anywhere and it is completely wireless." (I 22, ERP-consultant, study 1)

Barriers of learning in VR: Limitations of $360^{\circ}$ VR videos. From the above quotations, we conclude that an interactive VR scenario may be useful for individual learning if the complexity is aligned with the task. In contrast, all interviewees of the $360^{\circ}$ video mentioned that they could not see the possibility of using a $360^{\circ}$ video in the context of learning due to missing advantages in comparison to existing technologies.

"I think the video that I saw was not educational. [...] It would have been easier if I had read a book instead." (I 2, educator, study 2)

"But whether I had seen the video in the VR or on a monitor would not have made a difference." (I 3, student of IS, study 2)

Even the participants of the interactive scenario mentioned that they thought it made no sense to view a $360^{\circ}$ video in VR because the focus is on the video content which can also be watched on a computer screen. In this case, the device makes no difference.

"I don't think it makes sense to play a video in virtual reality. I have to fully concentrate on the video and for that I can just watch it on YouTube." (I 27, research associate, study 1)

Portability of virtual objects. Another difficulty lies in transferring virtual objects from virtual reality to actual reality. In traditional learning environments in which the individual learns in actual reality, activities such as note taking are easier to undertake. However, taking notes (e.g., to study for an exam) in virtual reality is clearly more difficult. Therefore, retrieving knowledge might be more difficult in actual reality compared to learning environments outside of virtual reality.

"There has to be the possibility to write and save your own notes, and to retrieve these notes from the program and use them privately." (I 15, production planners, study 1)

"I wouldn't know how I could study for exams with the help of virtual reality." (I 25, student of information systems, study 1)

Mixing up realities. If the learner has an especially intense or extensive VR experience actual and virtual reality could be mixed up in certain situations. There is a discrepancy between the "law of nature" in virtual reality compared to actual reality. The person can be confused if they have to apply the knowledge they acquired in VR to reality.

"Now when I look at something like that I always think I can zoom in on it. I think, if you play this for too long it could be dangerous. That somehow at some point - that you somehow confuse this VR world with the real world." [...] "And I see risks in the possibility of mixing up these worlds [the real world and virtual reality]. Moreover, you may walk in front of a car because you think you are still in this VR environment. I haven't been in there that long and somehow it's still a bit weird sometimes." (I 31, student of business administration, study 1)

"There could be the risk that you mix up reality with virtual reality." (I 21, student teacher, study 1) 
Boundaries of VR learning scenarios. In the interactive scenario, interviewees felt that other professions were appropriate for learning in immersive virtual reality but not their own one. In the interview, participants were asked if they thought it possible to learn how to change a tire in virtual reality and if it was possible for a nurse to practice taking blood in a virtual reality learning system. Whereas the technician pointed out how VR might be suitable for practicing how to take blood, the emergency paramedic underlined how it might be suitable for learning how to change a tire. However, both did not think that VR learning was appropriate for their own profession.

"In my opinion, you can learn to change a tire if you have practiced it in the virtual room several times. [...] I think it is not possible for a nurse to practice taking blood in a virtual environment because you have to be able to feel it and that's not possible in virtual reality. No, I do not think so." (I 7, emergency paramedic, study 1)

"A nurse can practice taking blood in a virtual environment, I think so." (I 10, electrical engineer, study 1)

This implies that the design of the virtual environment has to be carefully aligned with the requirements of the skill that has to be learned. Currently, VR systems are often less complex than actual reality because they fail to address physical or haptic feedback. However, in a task such as learning to take blood, haptic feedback with high granularity is crucial for learning.

The participants, especially in the interactive scenario, also mentioned this point of granularity. They stated that immersive VR was useful for practical learning and technical competences but not for gaining theoretical knowledge.

"As far as [regarding learning] visuals are concerned, yes, as far as techniques are concerned, yes, as far as memorizing is concerned, no." (I 2, student of teaching, study 1)

However, another interviewee disagreed by stating that even practical knowledge can be difficult to gain in virtual reality because current VR technology is unable to portray the complexity of reality. Thus, it may be possible to learn a sequence of steps of a skill but not the required fine motor skills. In this case, procedural knowledge, but not the sensory motor skills, can be addressed in a virtual reality learning system.

"I think it's risky to implement virtual reality in medical studies, theoretically you can learn how to operate on a person but you are working on a human being and you need the sensation. It is the same as retrieving knowledge from a book, you do not even know how a human 'works'. You also can't learn how to take blood in VR; you need the sensation and experience. [...] you can learn much easier in VR but it's all about applying it to reality." (I 30, practice nurse, study 1)

Implicit learning in the literature. After recognizing a theme of procedural learning in our empirical data, we searched the literature to arrive at a deeper understanding of implicit as well as explicit learning. In the literature on psychology, implicit learning is described as a unconscious process of learning that includes abstract knowledge [34]. In the literature on individual learning in immersive virtual reality, it is described as learning without conventional symbols, meaning learning is direct and personal. Therefore, the learner can solve abstract problems, whereby the concrete learning process takes part at a later time [47]. VR itself does not improve learning, it provides support or offers advantages when learning [5]. In contrast to implicit learning, explicit learning, also referred to as declarative learning, deals with the acquisition of knowledge through a consciousness act which can be recalled actively and deliberately [39].

Early studies have already investigated how knowledge acquired in virtual reality is applied to reality. In one study, participants were asked to lift and move cans in a virtual environment. However, there were no results on the transfer from the virtual to the real world because participants learned irrelevant skills, such as fine motor skills for lifting the can, that do not matter in the real world [20]. In turn, another study only examines sensory motor skills and shows a reliable transfer from virtual reality to reality [38]. If virtual objects and procedures are similar to real world activities the acquired skills can be applied in the real world fairly well. Similarly, Dinh et al. [9] conclude that visualization does not matter but tactile and auditory input as well as scents can strengthen implicit learning in VR. In contrast, Psotka [33] states that visualization in VR can enhance learning because individuals do not need to imagine visual content and, hence, have more cognitive resources for the actual task.

With regard to the perspective of a learner, such as being active or passive, recent studies show specific advantages of each perspective. For instance, if individuals are actively involved and in control in the VR environment, they acquire more knowledge compared to when they are passive [16]. Another study supports these findings and also states that the egocentric perspective, i.e., being active, is useful for tangible learning while an exocentric perspective, i.e., being passive, improves abstract and conceptual learning [7]. Roussou and Slater [40] provide similar results by showing the positive effect of interactivity (being active) on problem-solving skills while passive observing is helpful for learning concepts. 


\section{Discussion and Implications}

To answer our research question "What are the drivers and barriers of VR-HMDs in the context of individual learning?" we conducted an explorative case study in which we conducted interviews and a review of recent literature about VR and the context of learning. Literature already suggests several advantages of VR technology for individual learning. These advantages include immersion, i.e., a state of total engagement and involvement in a piece of technology or task, interactive scenarios in which a user can create, interact, or manipulate objects (including haptic feedback by provided controllers), visualization and depiction of abstract elements, applying practically infeasible methods, or breaking the boundaries of reality and laws of nature. In contrast, there are drawbacks of using VR in the context of learning, mainly related to simulator sickness and the way it manifests itself in motion sickness. Motion sickness occurs due to discrepancies between visual illusions and self-motion and hampers the immersion in and the focus on the learning task. As a consequence, motion sickness negatively affects learning in VR. In the following we will make suggestions for (future) research and developments of VR systems in the context of individual learning.

Our findings suggest that current VR systems provide an immersed virtual experience, which is also in line with recent literature [e.g., 19, 27, 44, 46, 47]. In this virtual space, an individual can experience something through diverse visualizations of abstract or real objects close to the real world and can precisely manipulate or create objects by using controllers. Our data also suggests that the high degree of mobility achieved through the use of $360^{\circ}$ videos is also an advantage. Anthes et al. [2] already mention the diversity of VR technologies. Our case study reveals the possibilities of high mobile learning environments, such as videos, combined with mobile VR systems (e.g. Google Cardboard or Samsung Gear VR). Learners can access content anyplace and probably anytime they wish. Consequently, we put forward our first proposition:

Proposition 1: VR technologies offer great potential for mobile application settings (independent of place and time).

In contrast, other findings suggests that VR $360^{\circ}$ learning videos are rather useless because they do not provide enough advantages in comparison to existing technologies, such as laptops and YouTube, or nontechnology solutions, such as books, or interactivity. Considering the $360^{\circ}$ video in our case study, we assume that this opinion could be influenced by the quality of the video. This experience might have been exhausting because each participant had to read the info panels in the video. In this case, one possible suggestion for the future would be to provide auditory content $[9,11]$ providing explanations, so a learner can concentrate on the actual step-by-step process better and will probably have an improved learning experience because it would feel more natural. Therefore, we put forward our second proposition:

Proposition 2: VR technologies should be enriched with auditory stimuli to make interactions appear more natural.

In accordance with recent research on VR and learning, we identified several barriers of VR for individual learning. These barriers manifest in different ways. First, as mentioned by Roussou et al. [39] and Roussou and Slater [40], declarative learning is suitable for more passive learning such as concepts and symbolisms. In turn, our findings suggest that VR systems, especially current VR technology [2], are appropriate for more low level learning. Therefore, we propose learning in VR as an implicit experience. The acquisition of procedural knowledge such as processes can benefit from using a VR system and interactivity. Moreover, VR technology allows its users to participate in more short-term interactions and tasks to learn about a process. From a practical point of view, e.g., employers who are establishing new processes in their companies, acquiring a skill that does not require a lot of time would be economically beneficial, particularly in terms of employees not wasting or spending too much time using a VR system. Moreover, another barrier arising from the use of current VR systems is the lack of transfer of objects with which a user can interact in the virtual space. For instance, if users take notes on content in the virtual space and want to reflect on these later, they cannot not just (physically or electronically) take these with them. Hence, learners in virtual environments are less able to acquire declarative knowledge in VR, for example when studying for exams. With this in mind, we put forward our third proposition:

Proposition 3: Learning in VR should focus on implicit outcomes and knowledge, such as procedures or processes.

Dinh et al. [9] suggest that solely focusing on visual effects in VR is not enough for learning, a learner needs tactile and auditory stimuli. Our research supports this view and suggests that sensory motor training helps a learner to apply knowledge from virtual reality to the real world [38]. According to our findings, current VR technologies do not provide sufficient haptic feedback to users in a virtual environment. This is also caused by the use of 
controllers rather than hands, and tactile feedback. Here, we conclude that the participant's view, referred to his or her own job, is transferable to another job but not to his or her own one. The belief that a VR system is useful for learning seems clear for each participant; but not for his or her own activities. In medicine, for instance, surgeons could learn how a human body works on a conceptual level. However, without a high level of sensitivity, they would not be able to really operate on a human. Similarly, a mechanic would not be able to really experience how to tighten a bolt. We therefore conclude that processes are much more complicated than current VR technologies are able to portray. However, with a high level of visualization and illustration of abstract or elements infeasible in the real world, VR can support users because they do not need to imagine visual content and have more cognitive resources for the learning task [33]. Consequently, we put forward our fourth proposition:

Proposition 4: To enable users to learn more complex contents and to acquire complex knowledge, $V R$ technologies should be enriched with tactile and sensory motor stimuli.

In their study, Konzak et al. [20] find that irrelevant interactions and skills lead to distractions from the actual task. If the task is not challenging enough, learners are easily distracted by unimportant elements. Considering our interactive scenario, this is important for learning cases, especially if the process or procedure individuals have to learn is too easy, so learners are easily distracted by the use of the controllers to interact in the virtual environment. As a consequence, such controllers (e.g., HTC Vive or controllers) can have a negative impact on the learning process because they can distract a learner from the actual task (for instance, a learner would focus more on how to handle the controller to move a parcel from one place to another than the procedure itself). Combing these arguments, our fifth proposition is:

Proposition 5: Irrelevant activities should not distract from individual learning in VR.

Immersion has been identified as a central positive factor relevant to learning in VR [e.g. 14, 26, 46, 47]. In turn, our findings suggest that being too immersed in the system might be problematic. In this case, a learner could mix up both realities. If virtual objects are perceived as real (this could be helpful for learning and applying skills in the real world [38]), users could be tempted to sit on virtual chairs, to rest on virtual tables, or to forget that they are wearing a HMD and move like they would in real life and collide with real objects. In turn, if subjects perceive real world objects as virtual they could believe that they cannot cause any harm. In a virtual environment, the virtual body can be affected but this will most likely not influence the real body (for instance, walking against a virtual door will lead to walking through the door). Consequently, interacting with real world objects would result in a (painful) accident. With this in mind, we put forward our sixth proposition:

Proposition 6: Immersion can be helpful for learning but being too immersed could lead to mixing up virtual and actual reality.

\section{Conclusions}

In this paper, we proposed drivers and barriers of current VR technologies for individual learning. We identified advantages, such as interactivity and mobility, but also limitations, particularly focusing on different types of use. For instance, using virtual objects in the real world (physically or electronically) still seems infeasible, the opportunity to confuse virtual and actual realities are facilitated by a high level of immersion, or the boundaries of VR learning scenarios are particularly apparent in explicit learning. Moreover, our findings suggest that implicit learning, such as procedural knowledge, should be pursued. In turn, current boundaries of VR systems include the inability to provide high sensitive haptic feedback when acquiring highly precise skills, that mechanics or surgeons would need for example. Consequently, future developments and research should take into account current limitations but also drivers of VR learning technologies to adequately address relevant topics and appropriate scenarios.

Acknowledgements. This article is funded by the project ELISE and the German Federal Ministry of Education and Research (BMBF, No. 16SV7512).

\section{References}

[1] Agarwal, R., and E. Karahanna, "Time flies when you're having fun: Cognitive absorption and beliefs about information technology usage", MIS quarterly, 2000, pp. 665-694.

[2] Anthes, C., R.J. Garcia-Hernandez, M. Wiedemann, and D. Kranzlmuller, "State of the art of virtual reality technology", IEEE (2016), 1-19.

[3] Benbasat, I., D.K. Goldstein, and M. Mead, "The Case Research Strategy in Studies of Information Systems", MIS Quarterly 11(3), 1987, pp. 369-386.

[4] Csikszentmihalyi, M., "Play and Intrinsic Rewards", In Flow and the Psychology of Discovery and Invention. HarperCollinsPublishers, 1996, 135-153. 
[5] Dalgarno, B., and M.J.W. Lee, "What are the learning affordances of 3-D virtual environments?", British Journal of Educational Technology 41(1), 2010, pp. 10-32.

[6] Darke, P., G. Shanks, and M. Broadbent, "Successfully Completing Case Study Research: Combining Rigour, Relevance and Pragmatism", Information Systems Journal 8(4), 2002, pp. 273-289.

[7] Dede, C., "Immersive Interfaces for Engagement and Learning”, Science 323(5910), 2009, pp. 66-69.

[8] Dede, C., J. Jacobson, and J. Richards, "Virtual, Augemented, and Mixed Realities in Education", In D. Liu, C. Dede, R. Huang and J. Richards, eds., Virtual, Augmented, and Mixed Realities in Education. Springer, 2017, 1-18.

[9] Dinh, H.Q., N. Walker, L.F. Hodges, C. Song, and A. Kobayashi, "Evaluating the Importance of Multi-Sensory Input on Memory and the Sense of Presence in Virtual Environments", Proceedings IEEE Virtual Reality (Cat. No. 99CB36316), (1999), 222-228.

[10] Eisenhardt, K.M., "Building Theories from Case Study Research", The Academy of Management Review 14(4), 1989, pp. 532-550.

[11] Garner, T., Sound in virtual reality and video games, Springer Berlin Heidelberg, New York, NY, 2017.

[12] Glaser, B., and A. Strauss, "The discovery ofgrounded theory", London: Weidenfeld and Nicholson 24(25), 1967, pp. 288-304.

[13] Gleasure, R., and J. Feller, "A Rift in the Ground: Theorizing the Evolution of Anchor Values in Crowdfunding Communities through the Oculus Rift Case Study", Journal of the Association for Information Systems 17(10), 2016.

[14] Goel, L., N.A. Johnson, I. Junglas, and B. Ives, "How Cues of What can be done in a Virtual World Influence Learning: An Affordance Perspective", Information \& Management 50(5), 2013, pp. 197-206.

[15] Hettinger, L.J., and G.E. Riccio, "Visually Induced Motion Sickness in Virtual Environments", Presence: Teleoperators and Virtual Environments 1(3), 1992, pp. 306310.

[16] James, K.H., G.K. Humphrey, T. Vilis, B. Corrie, R. Baddour, and M.A. Goodale, "'Active' and 'Passive' Learning of Three-Dimensional Object Structure within an Immersive Virtual Reality Environment", Behavior Research Methods, Instruments, \& Computers 34(3), 2002, pp. 383390.

[17] Kennedy, R.S., J. Drexler, and R.C. Kennedy, "Research in visually induced motion sickness", Applied Ergonomics 41(4), 2010, pp. 494-503.
[18] Keutel, M., B. Michalik, and J. Richter, "Towards Mindful Case Study Research in IS: A Critical Analysis of the Past Ten Years", European Journal of Information Systems 23(3), 2014, pp. 256-272.

[19] Khalifa, M., and N. Shen, "System Design Effects on Social Presence and Telepresence in Virtual Communities", Twenty-fifth International Conference on Information Systems, (2004).

[20] Kozak, J.J., P.A. Hancock, E.J. Arrthur, and S.T. Cchrysler, "Transfer of Training from Virtual Reality", Ergonomics 36(7), 1993, pp. 777-784.

[21] Lartigue, J., T. Scoville, and M. Pham, "Promoting K-8 Learning using Oculus Rift: Employing Virtual Reality to Increase Learning Outcomes in Elementary Biology", Association for the Advancement of Computing in Education (AACE) (2014), 1100-1105.

[22] LaViola, J.J., Jr., "A Discussion of Cybersickness in Virtual Environments”, SIGCHI Bull. 32(1), 2000, pp. 4756.

[23] Leong, P., "Role of Social Presence and Cognitive Absorption in Online Learning Environments", Distance Education 32(1), 2011, pp. 5-28.

[24] Lin, H., M. Chen, G. Lu, et al., "Virtual Geographic Environments (VGEs): A New Generation of Geographic Analysis Tool", Earth-Science Reviews 126, 2013, pp. 7484.

[25] Liu, D., R. Santhanam, and J. Webster, "Toward Meaningful Engagement: A Framework for Design and Research of Gamified Information Systems", MIS Quarterly 41(4), 2017, pp. 1011-1034.

[26] Magni, M., C. Paolino, R. Cappetta, and L. Proserpio, "Diving Too Deep: How Cognitive Absorption and Group Learning Behavior Affect Individual Learning", Academy of Management Learning \& Education 12(1), 2013, pp. 51-69.

[27] Martín-Gutiérrez, J., C.E. Mora, B. Añorbe-Díaz, and A. González-Marrero, "Virtual Technologies Trends in Education", EURASIA Journal of Mathematics, Science and Technology Education 13(1), 2017.

[28] Mills, S., and J. Noyes, "Virutal Reality: An Overview of User-related Design Issues", Interacting with Computers (11), 1999, pp. 375-286.

[29] Munafo, J., M. Diedrick, and T.A. Stoffregen, "The virtual reality head-mounted display Oculus Rift induces motion sickness and is sexist in its effects", Experimental Brain Research 235(3), 2017, pp. 889-901. 
[30] Müns, A., J. Meixensberger, and D. Lindner, "Evaluation of a Novel Phantom-based Neurosurgical Training System”, Surgical Neurology International 5, 2014.

[31] Ott, M., and L. Freina, "A Literature Review on Immersive Virtual Reality in Education: State of the Art and Perspectives", Conference proceedings of »eLearning and Software for Education « (eLSE), (2015), 133-141.

[32] Patton, M.Q., Qualitative Research, Wiley Online Library, 2005.

[33] Psotka, J., "Immersive Training Systems: Virtual Reality and Education and Training", Instructional Science 23(5-6), 1995, pp. 405-431.

[34] Reber, A.S., "Implicit learning of artificial grammars", Journal of verbal learning and verbal behavior 6(6), 1967, pp. 855-863.

[35] Reychav, I., and D. Wu, "Are Your Users Actively Involved? A Cognitive Absorption Perspective in Mobile Training", Computers in Human Behavior 44, 2015, pp. 335346.

[36] Reychav, I., and D. Wu, "Mobile Collaborative Learning: The Role of Individual Learning in Groups Through Text and Video Content Delivery in Tablets", Computers in Human Behavior 50, 2015, pp. 520-534.

[37] Rodríguez-Ardura, I., and A. Meseguer-Artola, "Flow in E-Learning: What Drives It and Why It Matters", British Journal of Educational Technology 48(4), 2017, pp. 899915.

[38] Rose, F.D., E.A. Attree, B.M. Brooks, D.M. Parslow, P.R. Penn, and N. Ambihaipahan, "Transfer of Training from Virtual to Real Environments", Virtual Reality, (1998), 6975.

[39] Roussou, M., M. Oliver, and M. Slater, "The Virtual Playground: An Educational Virtual Reality Environment for Evaluating Interactivity and Conceptual Learning", Virtual Reality 10(3-4), 2006, pp. 227-240.

[40] Roussou, M., and M. Slater, "Comparison of the Effect of Interactive versus Passive Virtual Reality Learning Activities in Evoking and Sustaining Conceptual Change", IEEE Transactions on Emerging Topics in Computing, 2017, p. $1-1$.

[41] Schafer, D.M., C. Carbonara, and M. Korpi, "Improved Virtual Reality Imaging and Gender: Factors Impacting Cybersickness", (2015).
[42] Schultze, U., "Embodiment and Presence in Virtual Worlds: A Review", Journal of Information Technology 25(4), 2010, pp. 434-449.

[43] Schultze, U., "Performing Embodied Identity in Virtual Worlds", European Journal of Information Systems 23(1), 2014, pp. 84-95.

[44] Seibert, J., and D.M. Shafer, "Control mapping in virtual reality: effects on spatial presence and controller naturalness", Virtual Reality 22(1), 2018, pp. 79-88.

[45] Sharples, S., S. Cobb, A. Moody, and J.R. Wilson, "Virtual reality induced symptoms and effects (VRISE): Comparison of head mounted display (HMD), desktop and projection display systems", Displays 29(2), 2008, pp. 58-69.

[46] Slater, M., and M.V. Sanchez-Vives, "Enhancing Our Lives with Immersive Virtual Reality", Frontiers in Robotics and AI 3, 2016.

[47] Slater, S., "Implicit Learning Through Embodiment in Immersive Virtual Reality", In D. Liu, C. Dede, R. Huang and J. Richards, eds., Virtual, Augmented, and Mixed Realities in Education. Springer, 2017, 19-34.

[48] Stone, R.J., "Blending the Best of the Real with the best of the Virtual: Mixed Reality Case Studies in Healthcare and Defence", In T. Jung and M.C. tom Dieck, eds., Augmented Reality and Virtual Reality. Springer Berlin Heidelberg, New York, NY, 2017, 277-294.

[49] Strauss, A., and J.M. Corbin, Basics of qualitative research: Grounded theory procedures and techniques., Sage Publications, Inc, 1990.

[50] Suh, K.-S., and Y.E. Lee, "The Effects of Virtual Reality on Consumer Learning: An Empirical Investigation", MIS Quarterly 29(4), 2005, pp. 673-697.

[51] Urquhart, C., and W. Fernandez, "Using grounded theory method in information systems: the researcher as blank slate and other myths", 2013, pp. 13.

[52] Wexelblat, A., ed., Virtual Reality Applications and Explorations, Academic Press Professional, Inc., San Diego, CA, USA, 1993.

[53] Yin, R., Case Study Research: Design and Methods, Sage Publications, 2013. 